Psychotherapeut 2020 $65: 465-474$ https://doi.org/10.1007/s00278-020-00465-3 Angenommen: 21. September 2020 Online publiziert: 8 . Oktober 2020 (c) Der/die Autor(en) 2020

\section{Therapeuten unterscheiden sich hinsichtlich der Therapieergebnis- se ihrer Patienten. Von zentraler Bedeutung für diesen sog. Thera- peuteneffekt sind wahrscheinlich die allgemeinen interpersonellen Fähig- keiten des Therapeuten. Eines der wenigen Instrumente, die Aspekte dieser Fähigkeiten messen, ist die Facilitative-interpersonal-skills(FIS)- Übung. In der FIS-Übung reagie- ren die Teilnehmenden verbal auf herausfordernde Therapiesituatio- nen, die in Videoclips nachgestellt wurden. Die audioaufgezeichneten Reaktionen werden von geschulten Ratern beurteilt.}

\section{Grundlagen}

\section{Der Therapeuteneffekt und Therapeutenfaktoren}

Therapeuten unterscheiden sich hinsichtlich der Therapieergebnisse ihrer Patienten. Ungefähr 5-9\% der Varianz des Therapieerfolgs können auf den mittlerweile gut belegten sog. Therapeuteneffekt zurückgeführt werden (Baldwin und Imel 2013; Crits-Christoph et al. 1991). Neben der Veränderung in der Symptomatik zeigt sich ein solcher Therapeuteneffekt auch für die Wahrscheinlichkeit eines Therapieabbruchs

Antje Gumz ${ }^{1,2} \cdot$ Merle Longley ${ }^{1}$ Lena Schestag ${ }^{1}$ Constance Hirschmeier ${ }^{1}$. Leonie Derwahl' ${ }^{\prime}$ Judith Weinreich ' Tobias Göttke' ${ }^{1}$. Friederike Höltermann ${ }^{1}$. Tobias Koch ${ }^{3} \cdot$ Katharina Freund' ${ }^{1}$ Margarita Geist ${ }^{1}$. Carina Schlipfenbacher ${ }^{1}$. Denise Kästner ${ }^{1,2}$

'Fachbereich Psychosomatik und Psychotherapie, Psychologische Hochschule Berlin, Berlin, Deutschland ${ }^{2}$ Klinik für Psychosomatische Medizin und Psychotherapie, Universitätsklinikum Hamburg-Eppendorf, Hamburg, Deutschland

${ }^{3}$ Institut für Psychologie, Friedrich-Schiller-Universität, Jena, Deutschland

\title{
Die „Facilitative interpersonal skills"-Übung - Messen therapeutischer Kompetenz mit der deutschsprachigen Version
}

(Gries et al. 2020; Zimmermann et al. 2017) und die Qualität der therapeutischen Allianz (Del Re et al. 2012). Auch wenn der generelle Therapeuteneffekt als gut nachgewiesen gilt, fehlt es bisher noch an Kenntnissen, welche Therapeutenfaktoren im Detail diese Unterschiede zwischen Therapeuten begründen (Gries et al. 2020; Heinonen und Nissen-Lie 2020a). Kenntnisse hierzu sind jedoch entscheidend für die Auswahl sowie für die Aus- und Weiterbildung von Psychotherapeuten und damit langfristig für erfolgreichere Psychotherapien.

Es ist ein zentrales Anliegen der Psychotherapie- und Ausbildungsforschung, Kenntnisse zu Therapeutenfaktoren, die den Therapeuteneffekt erklären, systematisch zu erfassen. Die bislang stärksten empirischen Hinweise zu Therapeutenfaktoren deuten auf die Bedeutsamkeit von allgemeinen interpersonellen Fähigkeiten und Eigenschaften wie Empathie und positive Wertschätzung hin (Heinonen und Nissen-Lie 2020b; Norcross und Lambert 2018).

Binder und Strupp (1997) arbeiteten heraus, dass es für Therapeuten schwierig sein kann, eine gute Therapiebeziehung aufrechtzuerhalten, v.a., wenn es zu feindseligen Interaktionen mit Patienten kommt („negative therapeutic process"). Eine aktuelle Metaanalyse (Eu- banks et al. 2018) zeigte, welche Bedeutung ein gelingender Umgang mit derartigen Verwicklungen in der Therapiebeziehung hat. Die Autoren fassten die Ergebnisse von bisher 11 vorliegenden Studien zum Effekt der Auflösung von Spannungen und Krisen in der Therapiebeziehung („alliance ruptures“) auf das Therapieergebnis zusammen. Der insgesamt moderate Zusammenhang bestand unabhängig von der therapeutischen Vorerfahrung, der Therapiedauer, der Therapiemethode, der Methode, , alliance ruptures" zu erfassen, und auch unabhängig davon, ob die Patienten eine diagnostizierte Persönlichkeitsstörung aufwiesen. Weitere Übersichtsarbeiten weisen darauf hin, dass der erfolgreiche Umgang mit schwierigen Gegenübertragungsreaktionen sowohl auf verbaler als auch nonverbaler Ebene zu besseren Therapieergebnissen beiträgt (Benecke et al. 2005; Hayes et al. 2018).

Einige Autoren konnten eine direkte Verbindung zwischen interpersonellen Fähigkeiten von Therapeuten, die von unabhängigen Ratern beurteilt wurden, und dem Therapieergebnis herstellen (Anderson et al. 2016a, 2016b; Schöttke et al. 2017). 


\section{Messen interpersoneller Fähigkeiten von Therapeuten}

Für eine standardisierte (vom individuellen Patienten unabhängige) Erhebung von Therapeutenfaktoren sind geeignete Messinstrumente erforderlich. Solche Instrumente sind nicht nur zur weiteren Forschung auf diesem Gebiet notwendig und nützlich. Auch für Auswahlverfahren sowie für die psychotherapeutische Aus- und Weiterbildung haben sie hohe praktische Relevanz. Die Reform der Psychotherapieausbildung verlangt eine deutlich stärkere berufspraktische Orientierung im Studium. Hierdurch werden solche Instrumente gefragter denn je sein. So sieht die neue Approbationsordnung u. a. vor, dass psychotherapeutische Kompetenzen im Rahmen von „objective structured clinical examinations" erfasst werden (OSCE; Bundesministerium für Gesundheit 2019). Dieses Prüfungsformat sowie generell die Arbeit mit simulierten und virtuellen Patienten kommt im Medizinstudium bereits langjährig zum Einsatz. Untersuchungen in diesem Kontext zeigen, dass die Arbeit mit simulierten und virtuellen Patienten eine nützliche und effektive Lernmethode sowohl als additive Ressource als auch im Vergleich zu herkömmlichen didaktischen Konzepten darstellt (Consorti et al. 2012). Allerdings deuten metaanalytische Ergebnisse ( $k=39$ Einzelstudien) auch darauf, dass OSCE-Bewertungen oft nicht reliabel sind und insbesondere die zwischen Prüfern übereinstimmende Erfassung von interpersonellen Fähigkeiten (vs. praktisch-medizinischen Kompetenzen) eine Schwierigkeit darstellt (Brannick et al. 2011).

Dem Wissen der Autoren des vorliegenden Beitrags nach existieren momentan nur 2 empirisch fundierte Instrumente zur Erhebung von interpersonellen Therapeutenfaktoren: 1 . „therapyrelated Interpersonal behaviors" (TRIB; Schöttke et al. 2017) und 2. FIS (Anderson et al. 2009; Gumz et al. 2018). Beim TRIB (Schöttke et al. 2017) wird die interpersonelle Kompetenz von Therapeuten erfasst, indem unabhängige $\mathrm{Ra}$ ter das Verhalten der Therapeuten in einer Gruppendiskussion beurteilen. Die FIS-Übung (Anderson et al. 2009; Gumz et al. 2018) steht im Zentrum vorliegender Studie.

\section{Die Facilitative-interpersonal- skills-Übung}

Anderson et al. (2009) entwickelten eine Methode, interpersonelle Fähigkeiten von Therapeuten zu messen, um die individuelle Bedeutung des Therapeuten und allgemeine therapeutische Wirkfaktoren therapieschulenunabhängig näher erforschen zu können. Mit diesen interpersonellen Fähigkeiten sind erlernte emotionale und interpersonelle Verhaltensmuster gemeint, die dazu verhelfen, mit schwierigen und komplexen interpersonellen Situationen effektiver umzugehen. Personen mit diesen Fähigkeiten sind in der Lage, ein breites Spektrum interpersoneller Botschaften wahrzunehmen, zu verstehen und zu vermitteln, und können gut zu Problemlösungen und neuen Verhaltensmustern motivieren (Anderson et al. 2009). Gemessen werden die interpersonellen Fähigkeiten in einer Übung mit simulierten Patienten ähnlich den OSCE. Bei der FISÜbung reagieren die Testteilnehmer verbal auf simulierte herausfordernde Therapiesituationen, die in Videoclips nachgestellt wurden. Die audioaufgezeichneten Reaktionen werden von geschulten Ratern im Hinblick auf folgende 8 interpersonelle Fähigkeiten beurteilt: die verbale Ausdrucksfähigkeit, die Fähigkeit, Hoffnung und positive Erwartungen zu vermitteln, die Fähigkeit, glaubwürdig und überzeugend zu sein, die emotionale Ausdrucksfähigkeit, die Fähigkeit, Wärme, Akzeptanz und Verständnis zu vermitteln, die Fähigkeit empathisch zu reagieren, die Bündnisfähigkeit sowie die Fähigkeit, vorhandene Spannungen und Krisen aufzugreifen (Gumz et al. 2018).

In weiterführenden Studien konnte die Autorengruppe zeigen, dass die mithilfe der FIS-Übung gemessenen interpersonellen Fähigkeiten von Therapeuten das Therapieergebnis der Patienten vorhersagen (Anderson et al. 2016a, 2016b).

Die Forschungsgruppe der Autoren des vorliegenden Beitrags entwickelte in Kooperation mit den Originalautoren eine deutschsprachige Version der FIS-
Übung. Dies umfasste die Produktion von 13 Videoclips sowie die Übersetzung und psychometrische Prüfung des zugehörigen FIS-Manuals (Gumz et al. 2018; Munder et al. 2019). In der ersten Untersuchung von 39 Ausbildungskandidaten konnten eine hohe Interrater-Reliabilität und interne Konsistenz gezeigt werden (Munder et al. 2019).

\section{Ziele}

1. Die deutschsprachige Version der FIS-Übung vorzustellen,

2. psychometrische und deskriptive Eigenschaften an einer größeren Stichprobe zu prüfen (Gesamtwert, Item-Werte, Interrater-Reliabilität, interne Konsistenz, Item-Schwierigkeiten und Mittwertunterschiede zwischen Clips und Clippositionen),

3. Zusammenhänge des FIS-Gesamtwerts mit Alter, Geschlecht und Ausbildungsstand der Durchführenden der FIS-Übung zu untersuchen.

Aus den Befunden werden Empfehlungen für den Einsatz der FIS-Übung in Forschung, Aus- und Weiterbildung abgeleitet.

\section{Material und Methoden}

\section{Studiendesign}

Die vorliegende Querschnittsuntersuchung ist Teil eines größeren Forschungsprojekts, das sich mit der deutschsprachigen Version der FIS-Übung beschäftigt. Neben den vorliegenden Auswertungen verfolgt das Projekt perspektivisch das Ziel, interpersonell kompetentes Verhalten näher zu charakterisieren sowie Zusammenhänge zwischen hilfreichen interpersonellen Fähigkeiten, anderen Merkmalen und Eigenschaften von Therapeuten sowie den Therapieergebnissen ihrer Patienten zu untersuchen.

\section{Ein- und Ausschlusskriterien und Rekrutierung}

An der Studie konnten Psychologiestudierende (Bachelor, Master) und Psychologen in psychotherapeutischer Ausbildung teilnehmen. Ausgeschlossen wur- 
Psychotherapeut 2020 -65:465-474 https://doi.org/10.1007/s00278-020-00465-3

(c) Der/die Autor(en) 2020

A. Gumz · M. Longley · L. Schestag · C. Hirschmeier · L. Derwahl · J. Weinreich · T. Göttke · F. Höltermann · T. Koch · K. Freund · M. Geist · C. Schlipfenbacher $\cdot$ D. Kästner

\section{Die „Facilitative interpersonal skills“-Übung - Messen therapeutischer Kompetenz mit der deutschsprachigen Version}

\section{Zusammenfassung}

Hintergrund. Sowohl für die Erforschung von Therapeuteneffekten als auch für die Aus- und Weiterbildung ist es von hoher Bedeutung, interpersonelle Fähigkeiten von Psychotherapeuten zu messen. Eines der wenigen dafür zur Verfügung stehenden Instrumente ist die Facilitative-interpersonalskills(FIS)-Übung.

Ziele der Arbeit. 1. Vorstellung der FIS-Übung, 2. Prüfung der psychometrischen und deskriptiven Eigenschaften und 3. Untersuchung der Zusammenhänge des FIS-Gesamtwerts mit Alter, Geschlecht und Ausbildungsstand der Testteilnehmer.

Material und Methoden. In die Querschnittsuntersuchung wurden Psychologiestudierende und Psychologen in psychotherapeutischer Ausbildung eingeschlossen. Die Teilnehmer sollten in der FIS-Übung auf herausfordernde Therapiesituationen, die in 13 Videoclips nachgestellt sind, verbal reagieren. Die audioaufgezeichneten Reaktionen wurden von je 3 geschulten Ratern eingeschätzt. Ergebnisse. Der durchschnittliche FISGesamtwert der 115 Studienteilnehmer (Alter: $M=31,97$ Jahre, $S D \pm 7,94$ Jahre; weiblich: $n=90,78,3 \%$; in psychotherapeutischer Ausbildung: $n=72$, $66,1 \%)$ betrug 3,24 (SD $\pm 0,42)$. Die Reliabilität erwies sich als gut (IntraklassenKorrelationskoeffizient [ICC]2 FIS-Gesamtwert $=0,69$; Cronbachs $a=0,958)$. Unter den Videoclips befanden sich 3 signifikant leichtere und 4 schwierigere. Über die Clips hinweg wurden sowohl ein Lern- als auch ein Ermüdungseffekt ersichtlich. Ausbildungskandidaten erzielten signifikant höhere Werte als Psychologiestudierende.

Diskussion. Die FIS-Übung erwies sich in einer größeren Stichprobe als reliabel. Es sollten mindestens 3 und maximal 9 Clips eingesetzt werden, die je nach Ausbildungsstand der Durchführenden und Clip-Schwierigkeit ausgewählt werden können. Die FIS-Übung ist zur standardisierten, empirisch fundierten Messung interpersoneller therapeutischer Fähigkeiten in Forschung und in der Aus- und Weiterbildung von großem Nutzen.

Schlüsselwörter

Professionelle Kompetenz $\cdot$ Interpersonelle Fähigkeiten · Ausbildungsforschung ·

Simulationspatienten. Psychotherapie

\section{The facilitative interpersonal skills exercise - Assessment of therapeutic competence with the German language version}

\section{Abstract}

Background. It is of great importance to measure interpersonal skills of psychotherapists for the research of therapeutic effects, training and further education. One of the few instruments available to measure these skills is the facilitative interpersonal skills (FIS) test. Objective. The aims of this study were 1) to present the FIS test, 2) to examine psychometric and descriptive properties and 3) to examine the associations between the total FIS score, age, gender and level of education of the performers.

Material and methods. Psychology students and psychologists in psychotherapy training were included in this cross-sectional study. In the FIS test the participants should verbally react to 13 patient videos that staged challenging therapy situations. Each of the audio-recorded reactions were assessed by three trained raters.

Results. The total FIS score of the 115 study participants (mean age $=31.97$ years, SD \pm 7.94 ; female $n=90,78.3 \%$; in psychotherapeutic training $n=72,66.1 \%$ ) averaged at 3.24 ( $S D \pm 0.42$ ). The reliability proved to be good (intraclass correlation coefficient, $\mathrm{ICC}_{\mathrm{FIS} \text { total value }}=0.69$; Cronbach's $a=0.958$ ). Among the video clips three were significantly less difficult and four clips were significantly more difficult. A learning effect and a fatigue effect became apparent throughout the clips. Trainee therapists achieved significantly higher scores than psychology students.
Conclusion. The FIS test proved to be reliable in a large sample. It was identified that a minimum of 3 and a maximum of 9 clips should be used during the test, which should be selected according to the level of training and clip difficulty. The FIS test is of considerable benefit for standardized and empirically based measurement of interpersonal therapist skills in research, training and further education.

\section{Keywords}

Professional competence $\cdot$ Interpersonal skills - Training research - Simulated patients . Psychotherapy den Personen, die über Vorwissen zur FIS-Übung verfügten. Die Rekrutierung der Studienteilnehmer fand über institutsinterne E-Mail-Verteiler der Psychologischen Hochschule Berlin (PHB) und der Berliner Akademie für Psychotherapie (BAP) sowie über Werbung für die Teilnahme in Seminaren und Vorlesungen und über Mundpropaganda statt.

\section{Datenerhebung}

Die Datenerhebung gliederte sich jeweils in 2 Abschnitte: 1. Beantwortung von Fragebogen (online, einschließlich soziodemografischer Informationen) und 2. FIS-Übung, einschließlich anschließender Selbsteinschätzung. Bei Interesse erhielten die Teilnehmer nach Abschluss ihrer Teilnahme Informationsmaterial zu den theoretischen und empirischen Hintergründen sowie eine individuelle Rückmeldung zu den Ergebnissen ihrer FISÜbung und ihrer Fragebogenantworten. 


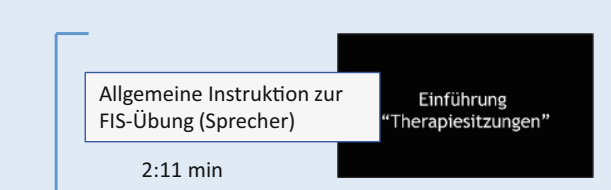

2:11 $\mathrm{min}$

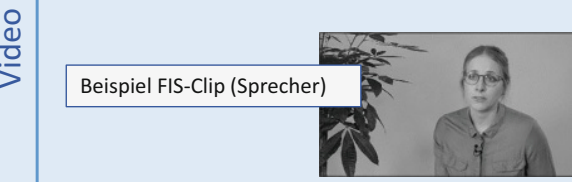

$1: 00-2: 19 \mathrm{~min}$

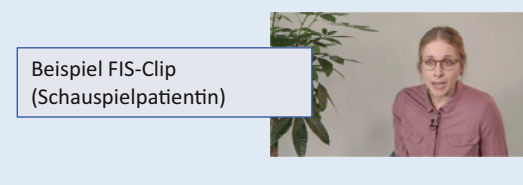

\begin{tabular}{ll|}
$\begin{array}{l}\text { Beispiel Therapeuten- } \\
\text { reaktion (Teilnehmer an FIS- } \\
\text { Übung) }\end{array}$ & REC \\
FIS-Gesamtwert für diese Therapeutenreaktion: 2,88
\end{tabular}
Die folgende Übung wird Ihnen die Möglichkeit geben, verschiedene herausfordernde Therapiesituationen exemplarisch kennenzulernen und zu erfahren, wie Sie spontan darauf reagieren. Die Aufgabe wird ca. 30 Minuten in Anspruch nehmen und besteht aus 13 Videosequenzen, auf welche Sie jeweils gebeten werden, mündlich zu reagieren. Ihre Aufgabe ist es, in diesen Situationen die Rolle des Therapeuten einzunehmen. [...] Versuchen Sie, sich vorzustellen, dass die Patientin direkt mit Ihnen als Therapeut spricht, und antworten Sie so, wie Sie denken, dass es am hilfreichsten wäre. Das Skript für die Videosequenzen ist an reale Therapiesituationen angelehnt. [...] Vor Beginn des Videos werden Sie jeweils einige Hintergrundinformationen zur Patientin erhalten. [...] Wenn das Video zu Ende ist, sind Sie an der Reihe, zu sprechen. Sie können [...] in Richtung des Bildschirms sprechen, ganz so, als ob Sie sich in einer Therapiesitzung mit der Patientin befinden würden.

Das ist Frau Schuster. Sie ist 26 Jahre alt und alleinerziehende Mutter eines 3-jährigen Jungen. Seit der Trennung vom Vater ihres Sohnes bewältigt sie die täglichen Anforderungen nur noch mit großer Anstrengung. Sie leidet unter häufigen Scham- und Schuldgefühlen und einem inneren Grundgefühl, „nicht richtig zu sein“. In der Therapie kommt sie seit einigen Sitzungen regelmäßig zu spät. Wenn das Video stoppt, sind Sie an der Reihe zu sprechen. Sagen Sie etwas zu Frau Schuster, als ob Sie die Therapeutin wären.

Ja, also ... ich weiß, dass ich wieder zu spät gekommen bin. Das ist mir echt peinlich, unangenehm, dass Sie 20 Minuten warten mussten. Ja, aber, irgendwie, ich provoziere das auch manchmal. Ich springe zu Hause rum, trödle, erledige schnell noch dies oder das. Ich provoziere, dass jemand wartet. Ich weiß auch nicht. Ich bin so unorganisiert. So bin ich eben. Naja. Ärgert Sie das? Ich wollte nur einfach mal fragen. Ich bin auch eigentlich froh, dass ich mir nicht mehr so den Kopf mache. Aber ich will nicht, dass Sie sich ärgern. Wenn Sie sagen würden, es ärgert mich, hätte ich echt mehr Ansporn, mehr pünktlich zu sein!

Ach, verstehe, Sie merken, dass Sie zu spät kommen ... und ja, was ich aus Ihrer Antwort entnehme, ist, das ich mich ärgern könnte, und Sie wissen nicht, ob ich mich ärgern würde. Es scheint mir, Sie wollen von mir erstmal wissen, wie es wirkt, wenn man zu spät kommt. Ist das denn so? Ähm ... Also Ärger, naja, Ich habe gedacht, dass das Ihre Stunde ist. Also, es ist ja so, dass das natürlich Ihre Zeit ist, die Sie hier zubringen und die geht Ihnen ja letztlich verloren. Sie müssen es wissen, ob Sie die Stunde nutzen wollen. Mein Ärger spielt ja hier eigentlich keine Rolle. Also ... beschreiben Sie mir doch bitte, was Sie damit verbinden, warum Sie meinen, dass ich mich ärgern könnte? Ist es, dass das ein Wunsch von Ihnen ist, dass jemand wartet? Also einer wartet und weiß nicht richtig, was los ist?

Abb. 1 ^ Ablauf der Facilitative-interpersonal-skills(FIS)-Übung

\section{Facilitative-interpersonal-skills- Übung}

\section{Aufbau und Ablauf}

Die FIS-Übung ist eine therapieschulenunabhängige Simulation, bei der die Teilnehmer spontan auf Äußerungen von Patienten so reagieren sollen, als wenn sie der Therapeut wären. Die Patientenäußerungen sind in kurzen Videoclips dargestellt. Zum Zeitpunkt der Entwicklung der deutschsprachigen Version der FIS-Übung existierten insgesamt 9 englischsprachige FIS-Clips. Diese 9 Clips wurden möglichst originalgetreu übersetzt und nachgedreht. Das Set wurde um zusätzliche 4 Clips mit typischen Szenen aus dem deutschsprachigen Therapiealltag erweitertet, um kulturspezifische Unterschiede zu berücksichtigen. Die deutschsprachige Version der FISÜbung besteht somit aus 13 Videoclips. Weitere Details zur Entwicklung finden sich in einer Vorarbeit (Munder et al. 2019).

Die Videoclips sind an reale, für Therapeuten besonders herausfordern- de Therapiesituationen angelehnt. Die meisten der Schauspielpatienten sind Kollegen, deren psychotherapeutische Kompetenz und Erfahrung es ermöglichte, sich hervorragend in die Patienten einfühlen zu können, einige sind professionelle Schauspieler. Vor jeder Patientenäußerung stellt ein Sprecher im Videoclip den jeweiligen Patienten kurz vor. In der aktuellen Untersuchung wurden alle 13 deutschsprachigen FISVideoclips (Frau Sel, Frau Klein, Frau Kaufmann, Herr König, Herr Dietz, Herr Busch, Herr Huth, Herr Beck, Herr Schneider, Frau Ziegler, Frau Schuhmann, Frau Bergmann, Herr Walther) in jeweils zufälliger Reihenfolge eingesetzt. Die Dauer der einzelnen Clips (Sprecher- und Patientenäußerung) beträgt zwischen 1:00 min und 2:19 min. Die Bearbeitung der gesamten FIS-Übung mit 13 Clips dauert ca. $30-50$ min (je nach Ausführlichkeit der Teilnehmerantworten). Während der Übung befindet sich der Teilnehmer allein und unbeobachtet in einem ruhigen Raum. Die Reaktionen des Teilnehmers auf die jeweiligen
Patientenäußerungen werden audioaufgezeichnet und im entsprechend nachfolgend beschriebenem Vorgehen ausgewertet.

Den Ablauf der FIS-Übung veranschaulicht Abb. 1 an einem Beispiel. Das in $\bullet$ Abb. 1 gezeigte Beispiel gehört nicht zu den 13 originalen FIS-Clips. Diese werden nicht publik gemacht, um für wissenschaftliche und Prüfungszwecke nutzbar zu bleiben.

\section{Auswertung}

Die Auswertung der audioaufgezeichneten Therapeutenreaktionen basiert auf dem FIS-Manual (Gumz et al. 2018). Darin werden die folgenden 8 FIS-Items unterschieden: 1. verbale Ausdrucksfähigkeit, 2. Hoffnung und positive Erwartungen, 3. Fähigkeit, glaubwürdig und überzeugend zu sein, 4. emotionale Ausdrucksfähigkeit, 5. Wärme, Akzeptanz und Verständnis, 6. Empathie, 7. Bündnisfähigkeit und 8. Aufgreifen von Spannungen und Krisen. Jede Therapeutenreaktion wird hinsichtlich dieser 8 Items auf einer 5-stufigen Likert-Skala (1-5) 
bewertet, wobei die 3 für einen mittleren Ausgangswert steht. Ausgehend davon werden niedrigere bzw. höhere Ausprägungen unter Berücksichtigung von verbalen Ankern für die Maximalwerte 1 und 5, die im Detail im Manual beschrieben sind, herunter- bzw. heraufgestuft. Dies stellt eine Modifikation zur amerikanischen Originalversion dar, in der verbale Anker für alle Werteausprägungen verwendet werden. Ziel dieser Modifikation war die Erreichung einer kontinuierlichen Skala. Aus dem Mittelwert über alle 8 FIS-Items wird ein FISGesamtwert gebildet. Die Eindimensionalität der Skala wurde in Vorarbeiten nachgewiesen (Munder et al. 2019). Alle FIS-Audioaufzeichnungen wurden von 3 geschulten Ratern unabhängig voneinander und ohne Kenntnis der Fragebogendaten oder soziodemografischen Informationen bewertet.

Das in $\bullet$ Abb. 1 gezeigte Beispiel wurde von drei geschulten Ratern wie folgt beurteilt: 1 . verbale Ausdrucksfähigkeit $(3,4,3), 2$. Hoffnung und positive Erwartungen $(3,3,3)$, 3. Fähigkeit, glaubwürdig und überzeugend zu sein $(4,3,3)$, 4. emotionale Ausdrucksfähigkeit (3, 3, 3), 5. Wärme, Akzeptanz und Verständnis $(3,3,2), 6$. Empathie $(3,3,4), 7$. Bündnisfähigkeit $(2,2,2)$ und 8 . Aufgreifen von Spannungen und Krisen (2, 2, 3).

\section{Rater und Rater-Schulung}

Die Schulung neuer Rater und fortlaufende Treffen des Rater-Teams fanden im wöchentlichen bis monatlichen Rhythmus statt. Die Rater-Schulung zielte auf ein genaues Verständnis des FIS-Manuals und der dargestellten Patientenfälle durch die Besprechung von diskrepanten Bewertungen in Übung-Ratings und die Arbeit mit Ankerbeispielen ab. Insgesamt waren 15 Rater ( $n=12$ weiblich, $n=3$ männlich) beteiligt, bei denen es sich um Psychologiestudierende $(n=2)$, Psychologen in psychotherapeutischer Ausbildung $(n=9)$ oder approbierte Psychotherapeuten $(n=1)$ handelte. In einem Fall wurden Ratings sowohl während des Studiums als auch in Ausbildung durchgeführt und in 2 Fällen sowohl in Ausbildung als auch nach der Approbation. Dabei waren sowohl psychodynamisch als auch verhaltensthera- peutisch orientierte Rater vertreten. Für die vorliegenden Auswertungen schätzte ein Rater im Mittel die Audioaufnahmen von 23 Teilnehmern ein.

\section{Datenanalyse}

In der Auswertung wurden zunächst deskriptive Maße zur Beschreibung der Stichprobe und der FIS-Werte ermittelt. Für die Interrater-Reliabilität wurden die Intraklassen-Korrelationskoeffizienten(ICC) 1 und 2 nach Bliese (2000) sowie $95 \%$-Konfidenzintervalle mithilfe des R-Pakets psychometric berechnet. Der ICC1 kann als Reliabilität der Gruppenmittelwerte (d.h. die mittlere RaterEinschätzung für eine Versuchsperson) für einen zufällig gezogenen Rater angesehen werden. Der ICC2 gibt die Reliabilität der Gruppenmittelwerte, gemittelt über alle zufällig gezogenen Rater, an. Die ermittelten ICC wurden auf Basis der aggregierten (d.h. gemittelten) Werte über alle Clips berechnet. Für die 13 FIS-Clips wurde deskriptiv jeweils die Item-Schwierigkeiten berechnet. Der Schwierigkeitsindex weist einen Wertebereich von 0 bis 100 auf, wobei höhere Werte für einfachere Clips stehen. Um anschließend potenzielle Unterschiede zwischen den Clips sowie Unterschiede zwischen Clippositionen (Reihenfolgeeffekte) zu untersuchen, wurden ,analyses of variance" (ANOVA) mit Messwiederholung gerechnet. Zur Ermittlung der internen Konsistenz wurde Cronbachs a verwendet. Zusammenhänge zwischen der abhängigen Variable FIS-Gesamtwert und den unabhängigen Variablen Alter, Geschlecht und Ausbildungsstand des Therapeuten wurden mithilfe linearer Regressionsanalysen untersucht. Die Analysen wurden mit $\mathrm{R}$ und SPSS vorgenommen.

\section{Ergebnisse}

\section{Stichprobencharakteristika}

Die 115 Studienteilnehmer waren im Mittel 31,97 Jahre (SD $\pm 7,94$ Jahre, Range: 19 bis 59 Jahre) und mit 78,3\% $(n=90)$ überwiegend weiblich (männlich: $n=25,21,7 \%)$. Die Mehrheit der Teilnehmer befand sich aktuell in einer
Partnerschaft $(n=54,47,0 \%)$ oder war verheiratet $(n=21,18,3 \%)$; etwa ein Drittel war alleinstehend $(n=38,33,0 \%)$, und 2 Teilnehmer waren geschieden $(1,7 \%)$. Der Großteil der Studienteilnehmer war bereits in psychotherapeutischer Ausbildung $(n=54,47,0 \%$ psychodynamisch orientiert; $n=22,19,1 \%$ verhaltenstherapeutisch). Etwas weniger als ein Drittel befanden sich im Psychologiestudium ( $n=19,16,5 \%$ Master, $n=16,13,9 \%$ Bachelor; $n=4$ fehlende Werte zur Ausbildung). Etwas mehr als die Hälfte der Teilnehmer $(n=59,51,3 \%)$ gaben an, über therapeutische Vorerfahrung $\mathrm{zu}$ verfügen ( $n=50,43,5 \%$ keine Vorerfahrung; $n=6,5,2 \%$ fehlende Werte). Dabei waren diese erfahrenen Teilnehmer im Mittel bereits 1,95 Jahre klinisch-psychologisch tätig $(S D \pm 2,25$ Jahre, Range: 1 bis 15 Jahre, einschließlich der praktischen Tätigkeit).

\section{FIS-Gesamtwert, FIS-Item-Werte und Interrater-Reliabilität}

Der durchschnittliche FIS-Gesamtwert in der untersuchten Stichprobe betrug 3,24 ( $S D \pm 0,42$, Range: 1,97-4,09). Die Durchschnittswerte in den FIS-Items schwankten zwischen 2,97 ( $S D \pm 0,58$, Range: $1,31-4,19)$ in dem Item „Aufgreifen von Spannungen und Krisen" und 3,36 ( $S D \pm 0,46$, Range: $1,82-4,28$ ) in der „emotionalen Ausdrucksfähigkeit“.

Die Interrater-Reliabilität für alle FISItem-Werte und für den FIS-Gesamtwert lag im guten bis exzellenten Bereich (•Tab. 1).

\section{Interne Konsistenz}

Die interne Konsistenz über alle FISClips betrug Cronbachs $a=0,958$. Wie - Tab. 2 zu entnehmen ist, wird ab einer Anzahl von 3 Clips mit einer 95\%igen Wahrscheinlichkeit eine interne Konsistenz über 0,80 erreicht.

\section{Item-Schwierigkeit und Mittelwertunterschiede für die FIS-Clips}

Für alle 13 FIS-Clips ergab sich eine mittlere Item-Schwierigkeit, wobei der leichteste Clip (Herr Busch) eine Item- 
Tab. 1 Deskriptive Statistik und Interrater-Reliabilität für den FIS-Gesamtwert und die FIS-Items

\begin{tabular}{|c|c|c|c|c|c|}
\hline & M & SD & Range & ICC1 (95\%-KI) & ICC2 (95\%-KI) \\
\hline Verbale Ausdrucksfähigkeit & 3,35 & $\pm 0,44$ & $1,83-4,28$ & $0,43(0,31-0,55)$ & $0,69(0,57-0,78)$ \\
\hline Hoffnung und positive Erwartungen & 3,22 & $\pm 0,37$ & $2,36-4,00$ & $0,61(0,50-0,70)$ & $0,82(0,75-0,87)$ \\
\hline Fähigkeit, glaubwürdig und überzeugend zu sein & 3,26 & $\pm 0,45$ & $1,78-4,31$ & $0,36(0,09-0,62)$ & $0,61(0,22-0,82)$ \\
\hline Emotionale Ausdrucksfähigkeit & 3,36 & $\pm 0,46$ & $1,82-4,28$ & $0,56(0,45-0,66)$ & $0,79(0,71,0,85)$ \\
\hline Wärme, Akzeptanz und Verständnis & 3,35 & $\pm 0,47$ & $2,00-4,11$ & $0,61(0,50-0,70)$ & $0,82(0,75-0,87)$ \\
\hline Empathie & 3,14 & $\pm 0,48$ & $1,79-4,15$ & $0,61(0,51-0,71)$ & $0,82(0,76-0,88)$ \\
\hline Bündnisfähigkeit & 3,25 & $\pm 0,48$ & $1,83-4,26$ & $0,66(0,56-0,74)$ & $0,85(0,79-0,89)$ \\
\hline Aufgreifen von Spannungen und Krisen & 2,97 & $\pm 0,58$ & $1,31-4,19$ & $0,68(0,60-0,76)$ & $0,86(0,81-0,90)$ \\
\hline FIS-Gesamtwert & 3,24 & $\pm 0,42$ & $1,97-4,09$ & $0,43(0,33-0,53)$ & $0,69(0,59-0,77)$ \\
\hline \multicolumn{6}{|c|}{$\begin{array}{l}\text { FIS „facilitative interpersonal skills“, M Mittelwert, SD Standardabweichung, ICC1 Intraklassen-Korrelationskoeffizient (Reliabilität der mittleren Ra- } \\
\text { ter-Einschätzungen für einen zufällig gezogenen Rater), ICC2 Reliabilität der mittleren Rater-Einschätzungen über alle zufällig gezogenen Rater, } \\
95 \%-K I 95 \% \text {-Konfidenzintervall } \\
\text { Die ICC2 wurden konservativ geschätzt und würden für alle Subskalen 0,90 überschreiten, falls die Beurteilungen nicht über alle Clips vor der Analys } \\
\text { gemittelt würden. Interpretation des ICC2 (Cicchetti 1994): <0,40: schlecht, 0,40-0,59: mäßig, 0,60-0,74: gut, 0,75-1,00: exzellent }\end{array}$} \\
\hline
\end{tabular}

\begin{tabular}{|c|c|c|}
\hline $\begin{array}{l}\text { Anzahl der } \\
\text { Clips }\end{array}$ & Cronbachs a & $95 \%$ KI \\
\hline 2 & 0,847 & $0,779-0,895$ \\
\hline 3 & 0,874 & $0,828-0,910$ \\
\hline 4 & 0,902 & $0,869-0,929$ \\
\hline 13 & 0,958 & $0,945-0,969$ \\
\hline \multicolumn{3}{|c|}{$\begin{array}{l}\text { FIS "facilitative interpersonal skills", KI Konfi- } \\
\text { denzintervall }\end{array}$} \\
\hline
\end{tabular}

Schwierigkeit von $60,14(S D \pm 11,66)$ und der schwierigste Clip (Herr Huth) eine Item-Schwierigkeit von 53,22 (SD $\pm 14,45)$ aufwies. Die FIS-Gesamtwerte in den einzelnen FIS-Clips unterschieden sich signifikant voneinander $(F(12,1224)=11,362 ; p<0,001$; partielles $\eta 2=0,100 ; n=103)$. Der Effekt der Clips betrug Cohens $f=0,33$ und entspricht einem mittleren Effekt. Die Bonferronikorrigierten Post-hoc-Tests deuten auf 3 besonders leichte FIS-Clips und 4 besonders schwierige FIS-Clips hin. Der leichteste Clip (Herr Busch) unterschied sich im FIS-Gesamtwert signifikant von 8 anderen FIS-Clips; für den zweitleichtesten Clip (Frau Kaufmann) ergaben sich 6 und für den drittleichtesten Clip (Frau Ziegler) 5 signifikante Unterschiede. Die Werte in den 4 schwierigsten Clips (Herr Huth, Herr Schneider, Herr Beck, Herr Dietz) lagen übereinstimmend unter den Werten in 5 anderen FIS-Clips (Frau Klein, Frau Kaufmann, Herr Busch, Frau Ziegler, Frau Schuh- mann). Für die übrigen Clips ergaben sich keine signifikanten Unterschiede.

\section{Reihenfolgeeffekte der FIS-Clips}

Da die einzelnen FIS-Clips in zufälliger Reihenfolge präsentiert wurden, ist es ebenfalls möglich, Reihenfolgeeffekte zu untersuchen. Entsprechend dem allgemeinen linearen Modells zeigte sich auch ein signifikanter Effekt der Clipposition $(\mathrm{F}(12,1224)=4,003$; $p>0,001$; partielles $\eta 2=0,038 ; n=103)$. Die Effektstärke beträgt Cohens $\mathrm{f}=0,20$ und spricht für einen schwachen Effekt. Betrachtet man jedoch die Kennwerte für den quadratischen Trend $(\mathrm{F}(12,1224)=21,686$; $p<0,001$; partielles $\eta 2=0,175$ ) ergibt sich eine Effektstärke von Cohens $\mathrm{f}=0,46$, die auf einen starken Effekt deutet. Der quadratische Trend und die Bonferronikorrigierten paarweise Vergleiche deuten sowohl auf einen Lern- als auch auf einen Ermüdungseffekt über die $13 \mathrm{Vi}$ deoclips hinweg hin (• Abb. 2). So war das Ergebnis im ersten FIS-Clip signifikant schlechter als das Ergebnis im 4., 5. und 7. Clip. Die Leistung im 10. und 13. Clip war wiederum signifikant schlechter als im 5. Clip.

\section{Zusammenhang mit Alter, Geschlecht und Ausbildungsstand der Durchführenden}

In der Regressionsanalyse (• Tab. 3) zeigte sich, dass Alter und Geschlecht keinen bedeutsamen Einfluss auf den
FIS-Gesamtwert haben. Im Gegensatz dazu erwies sich jedoch die Ausbildung als relevant. Studienteilnehmer, die aktuell im Bachelor- oder im Masterstudiengang Psychologie sind, weisen signifikant niedrigere FIS-Gesamtwerte im Vergleich zu den bereits graduierten Ausbildungsteilnehmern in tiefenpsychologischer Psychotherapie auf. Dabei erzielen Ausbildungsteilnehmer in Verhaltenstherapie fast identische FIS-Gesamtwerte wie ihre tiefenpsychologisch orientierten Kollegen. Das Gesamtmodell war signifikant und konnte etwa ein Viertel der Varianz (24,9\%) am FISGesamtwert aufklären.

\section{Diskussion}

\section{Zusammenfassung der Ergebnisse}

Die vorliegende Untersuchung hatte die Ziele, psychometrische Eigenschaften der deutschsprachigen Version der FISÜbung an einer größeren Stichprobe zu prüfen sowie Zusammenhänge zwischen dem FIS-Gesamtwert sowie dem Alter, Geschlecht und Ausbildungsstand zu untersuchen. Die Untersuchung ergab bei konservativer Schätzung gute bis exzellente Interrater-Reliabilitäten für den FIS-Gesamtwert und für alle FISItem-Werte sowie eine sehr hohe interne Konsistenz. Bei einer insgesamt mittleren Item-Schwierigkeit für alle Clips unterschieden sich die erzielten FIS-Gesamtwerte in einigen Clips signifikant voneinander. Die Ergebnisse deuten auf 


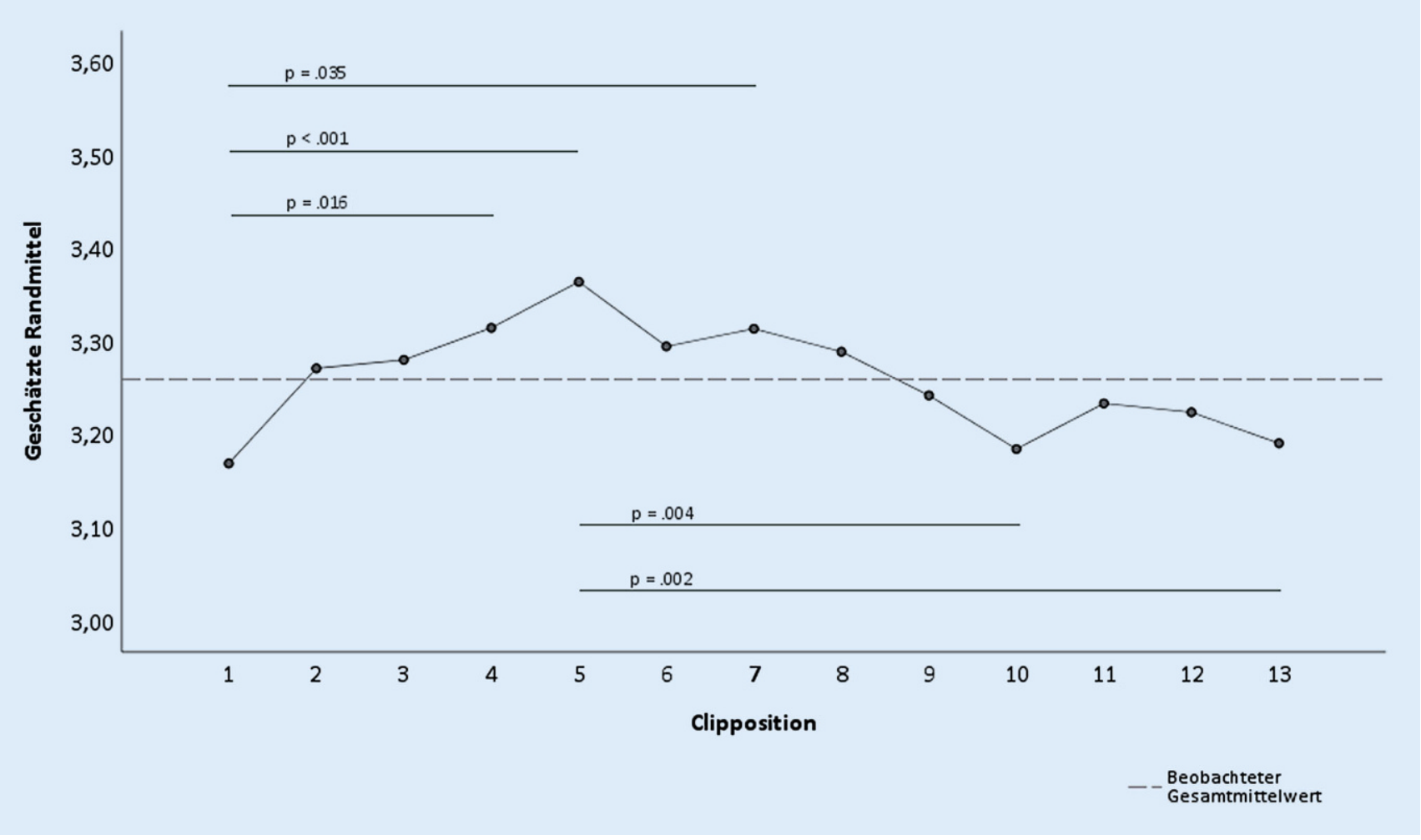

Abb. $2 \Delta$ Reihenfolgeeffekte der FIS-Clips

3 besonders leichte und 4 besonders schwierige FIS-Clips hin (signifikant höhere bzw. niedrigere FIS-Gesamtwerte). Darüber hinaus konnte ein Effekt der Clipposition verzeichnet werden, wobei über den Verlauf der Übung hinweg sowohl ein Lern- als auch ein Ermüdungseffekt ersichtlich wurde. Abschließend zeigte sich ein bedeutsamer Zusammenhang des FIS-Gesamtwerts mit dem aktuellen Ausbildungsstand.

\section{Psychometrische Güte}

Im Rahmen der OSCE ergeben sich oft keine hohen Übereinstimmungen zwischen unabhängigen Ratern bei der Bewertung von interpersonellen Fähigkeiten (Brannick et al. 2011). Im Gegensatz dazu konnte für die FIS-Übung bei einer sehr konservativen Berechnung (d.h. vorherige Aggregation der Beurteilungen über alle Clips) eine gute bis sehr gute Übereinstimmung erzielt werden. Die vorliegenden Ergebnisse bieten damit erste wichtige Anhaltspunkte dafür, dass die FIS-Übung für den Einsatz im Rahmen von Prüfungen gut geeignet ist. Tendenziell eher niedrigere Rater-Übereinstimmungen und breitere Konfidenzintervalle fanden sich für die Item-Werte zur „verbalen Ausdrucksfähigkeit“ und zur „Fähigkeit, glaubwürdig und überzeugend zu sein“. Für die „verbale Ausdrucksfähigkeit“ könnte überprüft werden, inwieweit objektive phonetische Marker eine reliablere und validere Alternative zur Rater-Einschätzung darstellen könnten. Das Item zur Glaubwürdigkeit wurde auch vom klinischen Eindruck der Rater her kritisch diskutiert und könnte perspektivisch konzeptuell weiterentwickelt werden. Mit Hinblick auf die erzielte Interrater-Reliabilität ist der Aufwand der Ratings zu berücksichtigen. Jeder Teilnehmer wurde von 3 Ratern eingeschätzt, und alle Rater absolvierten die zuvor beschriebene umfangreiche RaterSchulung. Interessant wäre hierbei zu betrachten, inwieweit Rater-Charakteristika die dyadische Interrater-Reliabilität beeinflussen. Für OSCE zu Kommunikationsfähigkeiten konnte ein solcher Effekt gezeigt werden (Mortsiefer et al. 2017). Signifikant höhere Interrater-Reliabilitäten wurden in Dyaden desselben Geschlechts, in Dyaden von 2 klinisch tätigen Ratern und in Dyaden mit viel OSCE-Erfahrung gefunden. Bei einer eher kleinen Rater-Gruppe $(n=15)$ und einer eher geringen Streuung in den interessierenden Variablen (z.B. Geschlecht, Ausbildungsstand) konnten in der aktuellen Untersuchung diese Einflüsse nicht betrachtet werden. Zukünftige Untersuchungen sollten dieser Fragestellung nachgehen, um weitere Empfehlungen für die Anwendung ableiten zu können.

\section{Anzahl von FIS-Clips}

Des Weiteren legen die Ergebnisse eine Kürzung der FIS-Übung nahe. Für diese Schlussfolgerung spricht einerseits der beobachtete Ermüdungseffekt, andererseits deutet die sehr hohe interne Konsistenz für den Gesamttest auf eine gewisse Redundanz hin. In der Studie wurden jedem Studienteilnehmer jeweils alle 13 existierenden FIS-Clips präsentiert.

Die Zahl der Clips könnte bei einem zukünftigen Einsatz reduziert werden. Für die amerikanische Version wurden zu Forschungszwecken 8 Clips verwendet (Anderson et al. 2016a; 2009). Die aktuellen Befunde zeigen, dass bereits 


\begin{tabular}{|c|c|c|c|c|c|c|c|c|}
\hline Prädiktoren & $\boldsymbol{\beta}$ & $95 \%-K I$ & SE & $\mathbf{t}$ & $p$ & $\mathbf{F}$ & $p$ & Adj. $R^{2}$ \\
\hline Alter & 0,101 & $\begin{array}{l}-0,003 \\
0,014\end{array}$ & 0,004 & 1,206 & 0,230 & 8,560 & $<0,001$ & 0,249 \\
\hline Geschlecht & $-0,002$ & $\begin{array}{l}-0,166 \\
0,163\end{array}$ & 0,083 & $-0,021$ & 0,983 & & & \\
\hline \multicolumn{9}{|c|}{ Ausbildungsstand } \\
\hline $\begin{array}{l}\text { Verhaltens- } \\
\text { therapie }\end{array}$ & $-0,004$ & $\begin{array}{l}-0,186 \\
0,177\end{array}$ & 0,092 & $-0,049$ & 0,961 & & & \\
\hline Master & $-0,299$ & $\begin{array}{l}-0,530 \\
-0,146\end{array}$ & 0,097 & $-3,486$ & 0,001 & & & \\
\hline Bachelor & $-0,461$ & $\begin{array}{l}-0,768 \\
-0,350\end{array}$ & 0,105 & $-5,302$ & $<0,001$ & & & \\
\hline \multicolumn{9}{|c|}{$\begin{array}{l}\text { FIS "facilitative interpersonal skills", KI Konfidenzintervall, SE Standardfehler. } n=111 \\
\text { Die Basiskategorie der Variable „Ausbildungsstand” ist die tiefenpsychologisch fundierte Psychothe- } \\
\text { rapie-Ausbildung } \\
\text { Alter und Ausbildung der Teilnehmenden sind nicht signifikant interkorreliert (Eta-Koeffizient: } \\
r=0,230, p=0,119)\end{array}$} \\
\hline
\end{tabular}

ab einer Zahl von 3 FIS-Clips mit einer hohen Wahrscheinlichkeit eine gute interne Konsistenz erreicht wird. Dabei ist zu berücksichtigen, dass die Leistung im allerersten FIS-Clip schlechter als in den darauffolgenden Clips ist. Dies wird als Lerneffekt interpretiert, der beispielsweise durch eine Gewöhnung an das Format der Übung eintritt. Der Lerneffekt wäre bei einem Einsatz für Prüfungen in dem Sinne zu beachten, dass jeder Prüfling vorab im Rahmen des Unterrichts mindestens einmal üben können sollte. $\mathrm{Ab}$ dem 10. aufeinander folgend absolvierten Clip muss von einem Abfall der Leistung ausgegangen werden. Es wird daher empfohlen, mindestens 3 und höchstens 9 Clips einzusetzen, um verlässliche Ergebnisse zu erzielen.

\section{Auswahl der FIS-Clips}

Um eine Vergleichbarkeit der Gesamtwerte bei der Verwendung von gekürzten FIS-Übungen zu gewährleisten, müssen die berichteten signifikanten Unterschiede zwischen den Clips beachtet werden. Dieser Befund widerspricht früheren, auf einer geringeren StichprobengröBe basierenden Beobachtungen (Munder et al. 2019). Dies betrifft im Forschungskontext beispielweise die Auswahl von Clips für wiederholte Messungen (z.B. Prä-post-Messungen). Bezogen auf eine Anwendung im Ausbildungskontext wird empfohlen, für Bachelorstudieren- de leichtere Clips und für fortgeschrittene Ausbildungskandidaten schwierigere Clips einzusetzen. Obwohl prinzipiell mittlere Item-Schwierigkeiten (Wert von ca. 50), wie sie für alle FIS-Clips vorlagen, zu bevorzugen sind, erscheint es sinnvoll, weitere deutlich leichtere bzw. schwierigere Clips (Item-Schwierigkeit: $<20$ schwer, $>80$ leicht) zu entwickeln, um im unteren und oberen Bereich noch genauer differenzieren zu können.

In zukünftigen Studien kann die Frage, welchen Teilnehmern welche Clips besonders schwer- oder leichtfallen, vertieft aufgegriffen werden. Hierzu gehört z. B. die Untersuchung von möglichen Unterschieden zwischen gleichund gegengeschlechtlichen FIS-PatientTherapeut-Dyaden. Im psychotherapeutischen Kontext existieren uneindeutige Befunde zum Einfluss der geschlechtlichen Passung von Patient und Therapeut (Gries et al. 2020). In der aktuellen Untersuchung zeigte sich jedoch, dass signifikant niedrigere Werte v. a. in Clips, die männliche Patienten zeigten, erzielt wurden. Da die Stichprobe der Studienteilnehmer überwiegend weiblich war (78,3\%), kann über einen Effekt der geschlechtlichen Passung spekuliert werden. Inwieweit dieser tatsächlich vorliegt oder eine Konfundierung mit anderen Variablen (z.B. feindseligeres, weniger zugewandtes Interaktionsangebot der dargestellten männlichen Patienten) hierfür ursächlich ist, sollte in weiteren Untersuchungen analysiert werden.

\section{Ethische Gesichtspunkte und Limitationen}

Der Einsatz der FIS-Übung erfordert, sich mit ethischen Fragen im Umgang mit den Teilnehmern vorab umfassend auseinanderzusetzen. Dies betrifft individuelle Rückmeldungen im Allgemeinen, die im Rahmen von Studien durch Pseudonymisierung, Passwortschutz und Involvierung mehrerer Studienmitarbeiter so gegeben werden sollten, dass keiner der Forschenden eine Zuordnung von FIS-Werten und identifizierenden Informationen (insbesondere Namen) vornehmen kann. Im Besonderen betrifft es Rückmeldungen von Kompetenzdefiziten. Das Erzielen vergleichsweise niedriger Werte in der FIS-Übung ist für die Teilnehmer u. U. emotional nicht leicht zu verarbeiten. Eine solche negative Rückmeldung berührt unmittelbar die berufliche Identität und den beruflichen Selbstwert und kann die Teilnehmer entmutigen. Dies spricht aus Sicht der Autoren dafür, die FIS-Übung relativ früh im Ausbildungsverlauf einzusetzen oder sie unterstützend für die Auswahl geeigneter Studierender zu nutzen. Allerdings ist zu berücksichtigen, dass positive individuelle Entwicklungen möglich und mit fortschreitender Ausbildung wahrscheinlich sind, und dass die Übung nicht das vollständige Potenzial bzw. alle Aspekte der therapeutischen Kompetenz abbildet. So fokussiert die FISÜbung in der Auswertung möglicherweise tendenziell stärker auf die verbale im Vergleich zur nonverbalen Kommunikation. Explizit und vorrangig wird die nonverbale Kommunikation im FIS durch die 2 Items zur verbalen und zur emotionalen Ausdrucksfähigkeit gemessen. Die anderen Items beziehen nonverbale Aspekte ein, erfassen jedoch in der Tendenz stärker verbale Aspekte, wobei beide Aspekte letztlich untrennbar miteinander verflochten sind (Pawelczyk 2011). Aufgrund der Verwendung von Audio- statt Videoaufzeichnungen bleiben des Weiteren potenziell bedeutsame Faktoren wie Mimik und Gestik (Benecke et al. 2005; Datz et al. 2019) bisher unberücksichtigt. Diese könnten jedoch theoretisch unter Abwägung der vorhan- 
denen datenschutzrechtlichen Nachteile integriert werden.

Darüber hinaus ist Psychotherapie stets ein interaktionelles Geschehen. In der FIS-Übung wird dies zugunsten der Standardisierung aufgehoben (Anderson et al. 2020). Dies ist eine wesentliche Grenze des Formats. Das FIS-Ergebnis könnte nicht nur von der therapeutischen Kompetenz, sondern auch von der Gewöhnung an dieses spezielle Testformat ohne unmittelbares Feedback von einem Gegenüber und von Ängsten vor Prüfungssituationen beeinflusst sein.

Bezogen auf die vorliegende Auswertung muss einschränkend erwähnt werden, dass es sich um eine Querschnittsuntersuchung handelt. Basierend auf den Ergebnissen erscheint es lohnenswert, längsschnittliche Untersuchungen über den Ausbildungsverlauf hinweg mit der FIS-Übung anzustellen, um mögliche Kompetenzzuwächse zu beobachten. Eine weitere Einschränkung der aktuellen Untersuchung liegt darin, dass ein Selektionsbias nicht ausgeschlossen werden kann. Die OSCE sind für die gesamte Kohorte verpflichtend, und die Ausschöpfung im Rahmen von Studien ist höchstwahrscheinlich hoch. Im Gegensatz dazu basierte die Teilnahme an der FIS-Übung auf Freiwilligkeit und könnte von individuellen Faktoren wie der Offenheit für neue Erfahrungen beeinflusst sein.

\section{Schlussfolgerung und Ausblick}

Die Entwicklung der Fähigkeiten von Therapeuten, interpersonell hilfreich mit herausfordernden Therapiesituationen umgehen zu können, ist therapieschulenunabhängig eine zentrale Aufgabe der Psychotherapieausbildung (Gumz 2020). Diesen Fähigkeiten wird künftig ein hoher und prüfungsrelevanter Stellenwert eingeräumt werden. Ausbildungsteilnehmern und auch rückblickend befragten Psychotherapeuten ist das praktische Lernen für die Entwicklung professioneller Fertigkeiten besonders wichtig (Nikendei et al. 2018; Orlinsky et al. 2001; Sonntag et al. 2009).

Die Ergebnisse der vorliegenden Untersuchung indizieren, dass die FISÜbung für den Einsatz in Forschung,
Aus- und Weiterbildung geeignet ist. Die Übung bietet die Möglichkeit, interpersonelle Fähigkeiten standardisiert und empirisch fundiert $\mathrm{zu}$ beurteilen. Dies kann dazu dienen, Therapeuteneffekte in der Forschung systematisch zu untersuchen und den Studierenden und Ausbildungsteilnehmern perspektivisch nach weiteren Analysen, z.B. Ermittlung von Bestehens- und Notengrenzen, Rückmeldungen zu ihrem aktuellen Leistungsstand zu geben oder Fähigkeiten im Rahmen von Prüfungen (z. B. OSCE) zu ermitteln.

Die deutschsprachige FIS-Übung kann ab Oktober 2020 auch online durchgeführt werden (www.psychotherapylab.de).

\section{Fazit für die Praxis}

- Die „Facilitative-interpersonalskills"(FIS)-Übung eignet sich, interpersonelle Fähigkeiten von Therapeuten standardisiert und empirisch fundiert zu beurteilen. Testteilnehmer reagieren verbal auf herausfordernde Therapiesituationen, die auf Videoclips nachgestellt wurden. Die Reaktionen werden audioaufgezeichnet und von geschulten Ratern eingeschätzt.

- Die FIS-Übung ist prinzipiell für den Einsatz in Forschung und Lehre geeignet. Im Hinblick auf die Psychotherapieausbildung könnte sie perspektivisch zur Auswahl von Studienbewerbern, für die „objective structured clinical examination" (OSCE) oder für Rückmeldungen zum individuellen Stand eingesetzt werden.

- Es wird empfohlen, für Bachelorstudierende leichtere Clips und für fortgeschrittene Ausbildungskandidaten schwierigere Clips einzusetzen.

- Mindestens 3 und maximal 9 Clips sollten genutzt werden. Bei der Auswahl der Clips sind die unterschiedlichen Schwierigkeitsgrade der Clips zu beachten.

\section{Korrespondenzadresse}

\section{Prof. Dr. Antje Gumz}

Fachbereich Psychosomatik und Psychotherapie, Psychologische Hochschule Berlin

Am Köllnischen Park 2, 10179 Berlin,

Deutschland

a.gumz@psychologische-hochschule.de

Danksagung. Wir bedanken uns bei allen Studierenden und Psychotherapeuten in Ausbildung, die an der FIS-Übung teilgenommen haben. Unser herzlicher Dank gilt auch allen Kollegen, Psychotherapeuten in Ausbildung und Schauspielerinnen, die an der Produktion der Video-Clips mitgewirkt haben, und dem Sprecher Dr. Rainer Erices. Wir bedanken uns ebenfalls bei unserer Kollegin Meike Hurrle, die uns erlaubt hat, die Aufnahmen mit ihr zur Veranschaulichung der FIS-Übung in $\bullet$ Abb. 1 im vorliegenden Beitrag zu nutzen.

Förderung. Die Studie zum deutschsprachigen FISTest wurde gefördert durch Drittmittel der Heigl Stiftung (20.05.2019; Projektleitung: Antje Gumz). Die Stiftung war nicht involviert in die Studienkonzeption, die Datenerhebung, die Datenanalyse oder die Ergebnisinterpretation.

Funding. Open Access funding enabled and organized by Projekt DEAL.

\section{Einhaltung ethischer Richtlinien}

Interessenkonflikt. A. Gumz, M. Longley, L. Schestag, C. Hirschmeier, L. Derwahl, J. Weinreich, T. Göttke, F. Höltermann, T. Koch, K. Freund, M. Geist, C. Schlipfenbacher und D. Kästner geben an, dass kein Interessenkonflikt besteht.

Alle beschriebenen Untersuchungen am Menschen oder an menschlichem Gewebe wurden mit Zustimmung der zuständigen Ethikkommission, im Einklang mit nationalem Recht sowie gemäß der Deklaration von Helsinki von 1975 (in der aktuellen, überarbeiteten Fassung) durchgeführt. Für die Studie liegt ein positives Ethikvotum der Ethikkommission der Psychologischen Hochschule Berlin vor (AZ:EK2020/06II). Von allen beteiligten Patienten liegt eine Einverständniserklärung vor.

Open Access. Dieser Artikel wird unter der Creative Commons Namensnennung 4.0 International Lizenz veröffentlicht, welche die Nutzung, Vervielfältigung, Bearbeitung, Verbreitung und Wiedergabe in jeglichem Medium und Format erlaubt, sofern Sie den/die ursprünglichen Autor(en) und die Quelle ordnungsgemäß nennen, einen Link zur Creative Commons Lizenz beifügen und angeben, ob Änderungen vorgenommen wurden.

Die in diesem Artikel enthaltenen Bilder und sonstiges Drittmaterial unterliegen ebenfalls der genannten Creative Commons Lizenz, sofern sich aus der Abbildungslegende nichts anderes ergibt. Sofern das betreffende Material nicht unter der genannten Creative Commons Lizenz steht und die betreffende Handlung nicht nach gesetzlichen Vorschriften erlaubt ist, ist für die oben aufgeführten Weiterverwendungen des Ma- 
terials die Einwilligung des jeweiligen Rechteinhabers einzuholen.

Weitere Details zur Lizenz entnehmen Sie bitte der Lizenzinformation auf http://creativecommons.org/ licenses/by/4.0/deed.de.

\section{Literatur}

Anderson T, Crowley ME, Himawan L, Holmberg JK, Uhlin BD (2016a) Therapist facilitative interpersonal skills and training status: a randomized clinical trial on alliance and outcome. Psychother Res 26(5):511-529. https://doi.org/10.1080/ 10503307.2015.1049671

Anderson T, Finkelstein JD, Horvath SA (2020) The facilitative interpersonal skills method: difficult psychotherapy moments and appropriate therapist responsiveness. Couns Psychother Res. https://doi.org/10.1002/capr.12302

Anderson T, McClintock AS, Himawan L, Song $X$, Patterson CL (2016b) A prospective study of therapist facilitative interpersonal skills as a predictor of treatment outcome. J Consult Clin Psychol 84(1):57-66. https://doi.org/10.1037/ ccp0000060

Anderson T, Ogles BM, Patterson CL, Lambert MJ, Vermeersch DA (2009) Therapist effects: facilitative interpersonal skills as a predictor of therapist success. J Clin Psychol 65(7):755-768. https:// doi.org/10.1002/jclp.20583

Baldwin SA, Imel ZE (2013) Therapist effects: findings and methods. In: Lambert MJ (Hrsg) Bergin and Garfield's handbook of psychotherapy and behavior change, Bd. 6, S258-297

Benecke C, Peham D, Bänninger-Huber E (2005) Nonverbal relationship regulation in psychotherapy. Psychother Res 15(1-2):81-90

Binder JL, Strupp HH (1997) "Negative process": a recurrently discovered and underestimated facet of therapeutic process and outcome in the individual psychotherapy of adults. Clin Psychol Sci Pract 4(2):121-139

Bliese PD (2000) Within-group agreement, nonindependence, and reliability: implications for data aggregation and analysis multilevel theory, research, and methods in organizations: foundations, extensions, and new directions. Jossey-Bass, San Francisco, S 349-381

Brannick MT, Erol-Korkmaz HT, Prewett M (2011) A systematic review of the reliability of objective structured clinical examination scores. Med Educ 45(12):1181-1189. https://doi.org/10.1111/j. 1365-2923.2011.04075.x

Bundesministerium für Gesundheit (2019) Entwurf einer Approbationsordnung für Psychotherapeutinnen und Psychotherapeuten (PsychTh-ApprO). https:// www.bundesgesundheitsministerium.de/ fileadmin/Dateien/3 Downloads/Gesetze und_Verordnungen/GuV/P/PsychThApprO_ RefE.pdf.Zugegriffen:24.06.2020

Cicchetti DV (1994) Guidelines, criteria, and rules of thumb for evaluating normed and standardized assessment instruments in psychology. Psychol Assess 6(4):284-290. https://doi.org/10.1037/ 1040-3590.6.4.284

Consorti F, Mancuso R, Nocioni M, Piccolo A (2012) Efficacy of virtual patients in medical education: a meta-analysis of randomized studies. Comput Educ 59(3):1001-1008.https://doi.org/10.1016/ j.compedu.2012.04.017
Crits-Christoph P, Baranackie K, Kurcias J, Beck A, Carroll K, Perry K, ..., Gallagher D (1991) Metaanalysis of therapist effects in psychotherapy outcome studies. Psychother Res 1:81-91

Datz F, Wong G, Löffler-Stastka H (2019) Interpretation and working through contemptuous facial micro-expressions benefits the patient-therapist relationship. IJERPH 16:4901

Del Re AC, Flückiger C, Horvath AO, Symonds D, Wampold BE (2012) Therapist effects in the therapeutic alliance-outcome relationship: a restricted-maximum likelihood meta-analysis Clin Psychol Rev 32(7):642-649. https://doi.org/ 10.1016/j.cpr.2012.07.002

Eubanks CF, Muran JC, Safran JD (2018) Alliance rupture repair: a meta-analysis. Psychotherapy 55(4):508-519. https://doi.org/10.1037/ pst0000185

Gries S, Longley M, Kästner D, Gumz A (2020) Therapeutenmerkmale und Dropout - Ein systematisches Review der letzten 20 Jahre. Psychotherapeut. https://doi.org/10.1007/s00278-020-00454-6

Gumz A (2020) Umgang mit Spannungen und Krisen in der Therapiebeziehung. Psychotherapeut 65(2):119-132. https://doi.org/10.1007/ s00278-020-00400-6

Gumz A, Munder T, Schlipfenbacher C, Toussaint $K$, Warmuth M (2018) Messen interpersoneller therapeutischer Kompetenz. FIS-Manual, deutsche Version. https://www.researchgate. net/publication/330523365_FIS-Manualdeutsche-Version.Zugegriffen:26.08.2020

Hayes JA, Gelso CJ, Goldberg S, Kivlighan DM (2018) Countertransference management and effective psychotherapy: meta-analytic findings. Psychotherapy 55(4):496-507. https://doi.org/ $10.1037 /$ pst0000189

Heinonen E, Nissen-Lie HA (2020a) The professional and personal characteristics of effective psychotherapists: a systematic review. Psychother Res 30(4):417-432. https://doi.org/10.1080/ 10503307.2019.1620366

Heinonen E, Nissen-Lie HA (2020b) The professional and personal characteristics of effective psychotherapists: a systematic review. Psychother Res 30(4):417-432. https://doi.org/10.1080/ 10503307.2019.1620366

Mortsiefer A, Karger A, Rotthoff T, Raski B, Pentzek M (2017) Examiner characteristics and interrater reliability in a communication OSCE. Patient Educ Couns 100(6):1230-1234.https://doi.org/ 10.1016/j.pec.2017.01.013

Munder T, Schlipfenbacher C, Toussaint K, Warmuth M Anderson T, Gumz A (2019) Facilitative interpersonal skills performance test: psychometric analysis of a German language version. J Clin Psychol 75(12):2273-2283. https://doi.org/10. 1002/jclp.22846

Nikendei C, Bents H, Dinger U, Huber J, Schmid C, Montan I, Safi A et al (2018) Erwartungen psychologischer Psychotherapeuten zu Beginn ihrer Ausbildung: Qualitative Interviewstudie mit Vergleich von Verhaltens- und tiefenpsychologisch fundierter Psychotherapie. Psychotherapeut 63(6):445-457. https://doi.org/10.1007/ s00278-018-0312-2 ([Expectations of psychological psychotherapists at the beginning of training. Qualitative interview study with comparison of behavioral and depth psychologybased psychotherapy.])

Norcross JC, Lambert MJ (2018) Psychotherapy relationships that work III. Psychotherapy 55(4):303-315. https://doi.org/10.1037/ pst0000193
Orlinsky DE, Botermans JF, Rønnestad MH (2001) Towards an empirically grounded model of psychotherapy training: four thousand therapists rate influences on their development. Aust Psychol 36(2):139-148. https://doi.org/10. 1080/00050060108259646

Pawelczyk J (2011) Talk as therapy: Psychotherapy in a Linguistic Perspective. De Gruyter Mouton, Berlin, Boston

Schöttke H, Flückiger C, Goldberg SB, Eversmann J, Lange J (2017) Predicting psychotherapy outcome based on therapist interpersonal skills: A fiveyear longitudinal study of a therapistassessment protocol. Psychother Res 27(6):642-652. https:// doi.org/10.1080/10503307.2015.1125546

Sonntag A, Glaesmer H, Barnow S, Brähler E, Fegert J, Fliegel S, Strauss B et al (2009) Die Psychotherapeutenausbildung aus Sicht der Teilnehmer. Ergebnisse einer Ausbildungsteilnehmerbefragung im Rahmen des Forschungsgutachtens. Psychotherapeut 54:427-436. https://doi.org/ 10.1007/s00278-009-0704-4

Zimmermann D, Rubel J, Page AC, Lutz W (2017) Therapist effects on and predictors of nonconsensual dropout in psychotherapy. Clin Psychol Psychother 24(2):312-321. https://doi. org/10.1002/cpp.2022 